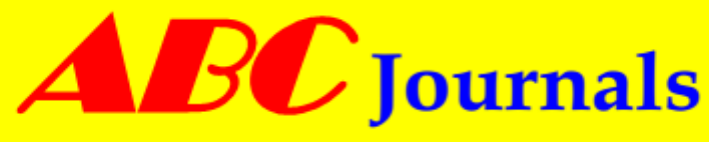

A new domain in research publishing

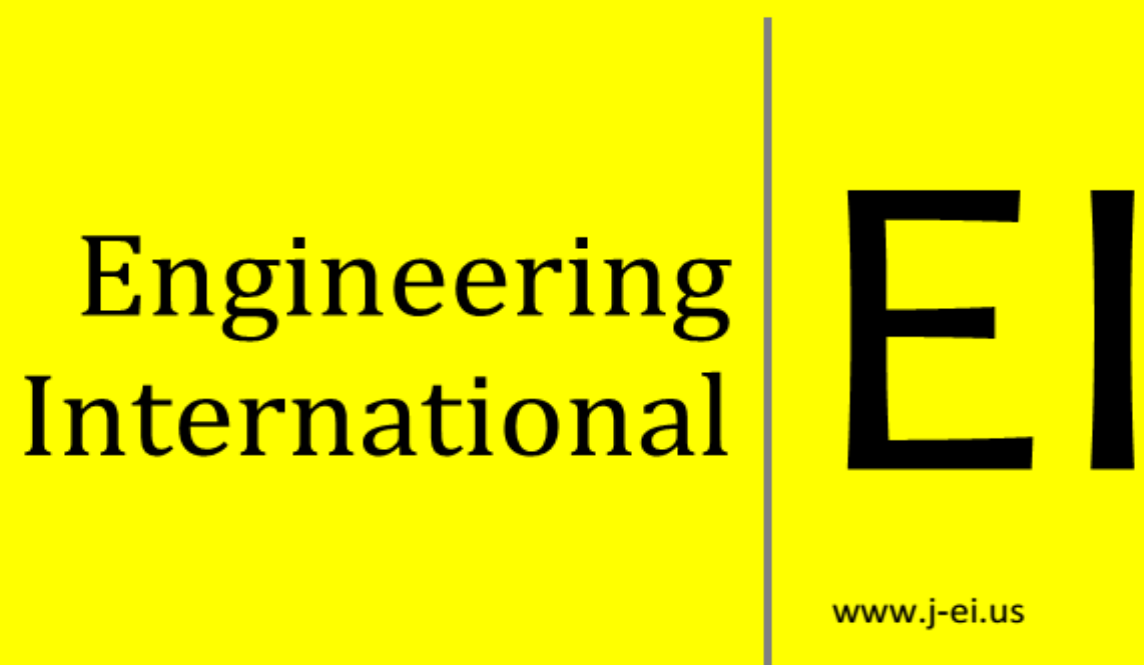

Asian Business Consortium 


\title{
Electrical Characterization and Doping Uniformity Measurement during Crystalline Silicon Solar Cell Fabrication Using Hot Probe Method
}

Nahid Akter', Md. Abul Hossion ${ }^{2}$, Mahbubul Hoq ${ }^{3}$, Sardar Masud Rana ${ }^{4}$, Md Anzan-Uz-Zaman ${ }^{5}$, Md. Nasrul Haque $\mathrm{Mia}^{6}$, Md. Alamgir Kabir ${ }^{7}$ \& Zahid Hasan mahmood ${ }^{8}$

1,2,7,8 Applied Physics, Electronics and Communication Engineering, Dhaka University, Bangladesh

3,4,5,6 Institute of Electronics, Atomic Energy Research Establishment (AERE), Dhaka, Bangladesh

\begin{abstract}
The parameters of crystalline semiconductor such as types of semiconductor, uniformity of impurity concentration of doped wafer, majority charge carrier concentration, sheet resistivity of doped wafer surface play an important role in solar cell fabrication process during emitter diffusion, that is the most critical step. In this paper, we have used a low cost in house made hot probe measurement setup. A hot plate was used to heat up the wafer up to $100^{\circ} \mathrm{C}$. Two k-type thermocouples were placed simultaneously in contact with the hot and cold surface of the wafer to measure the temperature in situ for both hot and cold probe. We have used two copper probes with a voltmeter connected to measure the potential difference (thermoelectric voltage) between two probes for various temperatures up to $100^{\circ} \mathrm{C}$ with an interval of $10^{\circ} \mathrm{C}$. We have taken measurement for commercial silicon wafer (thickness $200 \mu \mathrm{m}$ ) and one side polished 4 inch diameter Si wafer (thickness $660 \mu \mathrm{m}$ ) to determine the wafer type (n-type or p-type). We also calculated thermopower or Seebeck coefficient from the voltage vs. time curve, that is constant for particular substrate. As a process monitoring tool for solar cell fabrication process, after n-type diffusion using $\mathrm{POCl} 3$ on p-type silicon wafer of thickness $200 \mu \mathrm{m}$, we have done wafer mapping that gives us the information of doping uniformity over the whole surface of wafer both front and back side
\end{abstract}

Keywords: Hot probe, Seebeck effect, Thermal conductivity, Wafer mapping,

\section{INTRODUCTION}

Silicon has a crystalline structure and it has grown under controlled conditions to exhibits certain desired and predictable characteristics. As a starting material used for solar cell fabrication process called wafer or substrate, so that, it is necessary to characterize the substrate. Doped Si becomes either p or n-type. Dopant atoms could be either "donors" or "acceptors." Donors increase the electron concentration in the silicon, whereas acceptors 
increase the hole concentration. To characterize the substrate for semiconductor fabrication process, it is necessary to know the dopant type. Hot probe measurement is one of the ways to determine whether the wafer is p-type doped or n-type doped (Axelevitch 2013).

\section{Hot Probe Theory}

The simple "hot-probe" experiment consists of a soldering iron and a standard voltmeter to distinguish between n-type and p-type semiconductors. A semiconductor wafer is contacted with a "hot" probe such as a heated soldering iron and room temperature "cold" probe. Hot probe is connected to the positive terminal of the meter and the cold probe is connected to the negative terminal and then it is measured the direction of current flow between the two probes. Positive current reading or positive voltage reading on the meter determines the material is n-type and the reverse is p-type. Current flows from the hot probe to the cold probe for the n-type wafer, while current flows in the opposite direction for the p-type wafer. The explanation is that the thermally excited majority free charged carriers are diffused within the semiconductor from the hot probe to the cold probe. A temperature gradient generates potential difference between probes called Seebeck voltage. For n-type, electrons diffuse from hot to cold probe throughout the sample setting up an electric field that produces a potential difference and then detected by voltmeter. Actually, electrons flow through the cold probe (negative) and current (hole) flows in the opposite direction with the hot probe positive, so that the meter reading is positive. Similarly, for p-type, holes flow through the cold probe (negative) with the hot probe positive, so that the meter reading is negative (Keenan 1971; ASTM Standard 1996). If the current meter has zero resistance with ignoring the (small) thermoelectric effect in the metal wires, one can justify that the Fermi energy does not vary throughout the material. The corresponding energy band diagram illustrates the specific case in which the temperature variation causes a linear change of the conduction band energy as measured relative to the Fermi energy. As the effective density of states decreases with decreasing temperature, the conduction band energy decreases with decreasing temperature yielding an electric field which causes the electrons to flow from higher to lower temperature. The same reasoning reveals that holes in a p-type semiconductor will also flow from higher to the lower temperature (Axelevitch 2013).

\section{EXPERIMENTAL DETAILS}

Here, Image 1 shows a low cost in house made hot probe measurement setup. A hot plate was used to heat up the wafer where a temperature controller was used to provide the predefined temperature. In this procedure, we had to set the temperature first in the temperature controller then pressing 'Run' and at the same time a regulatory knob of the hot plate was set to low to high and then waited for few minutes to reach the predefined temperature. Portion of $\mathrm{Si}$ wafer was placed on the hot plate where the rest was placed on a nonconductive base.

Two k-type thermocouples were placed simultaneously in contact with the hot and cold surface of the wafer to measure the temperature in situ for both hot and cold probe.

The wafer began to heat and thermally generated carriers started to diffuse through it from hot to cold portion. Two copper probes were connected with a voltmeter to measure the potential difference (thermo-electrical voltage) between two probes for various temperatures. The red probe (positive) of the voltmeter was attached on the hot side and black probe (negative) was attached on the cold side of the wafer. 


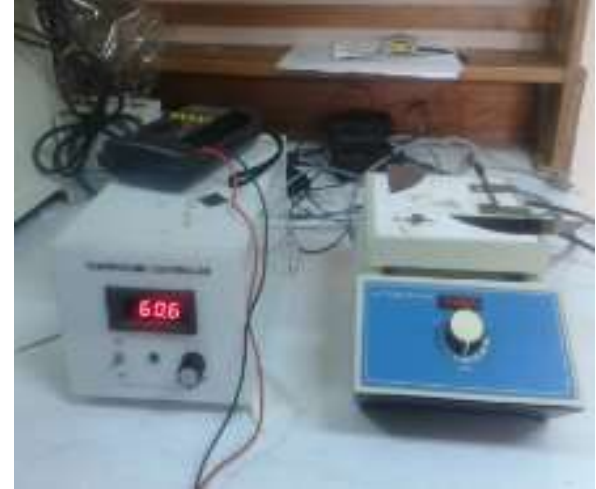

(a)

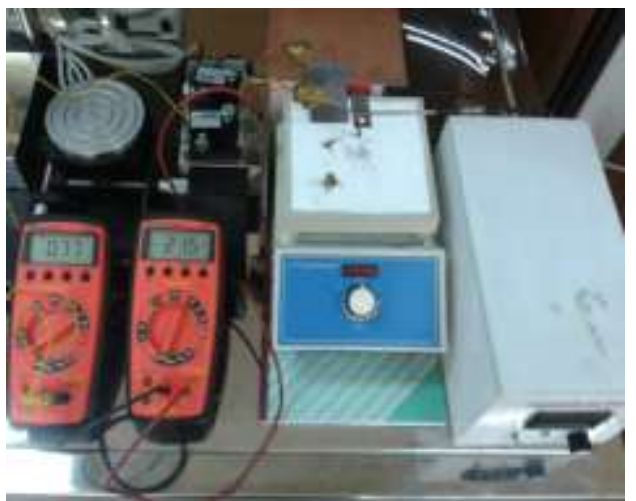

(b)

Image 1. (a) Conventional hot plate with temperature controller (b) Hot probe measurement experimental set up.

The thermally generated charge carriers mainly diffused from the hot side to the cold side and were collected by the black probe. Finally, the voltmeter showed (+/-) voltage due to temperature gradient potential difference.

\section{RESULTS}

Figure 1 and 2 shows the hot probe characteristic curves measured in bulk crystalline Silicon samples of different thickness for different temperatures of hot electrode. These curves demonstrate positive or negative voltage measured between the two electrodes. If the voltage is positive, the sample is n-type and if the voltage is negative, the sample is p-type. By increasing the hot probe temperature, the measured voltage between electrodes is also increased. All curves are similar to each other. Three different regions may be distinguished in the obtained characteristics. A steep rising at the first part, then fast decreasing to a steady state which continues decreasing slowly and decreasing sharply after removing the hot plate from the electrode. The slope of the voltage decreased in the middle part of the curves is influenced by the cold electrode temperature which is increased due to thermal conductivity of the crystalline $\mathrm{Si}$. The initial growth and final decaying are explained by charge carrier generation and recombination. Thermo-power can be obtained by steady state voltage divided by temperature, and it is constant for each sample.

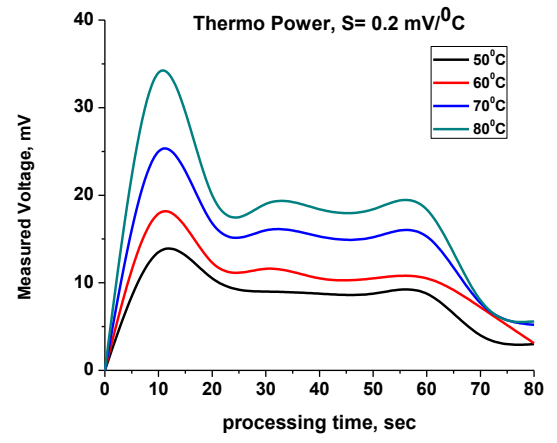

(a)

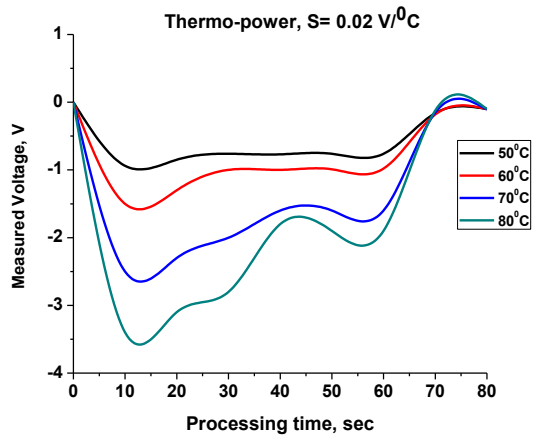

(b)

Figure 1. Characteristic curves for (a) n-type one side polished Si $(660 \mu \mathrm{m})$ sample (b) p-type one side polished $\mathrm{Si}(660 \mu \mathrm{m})$ sample 
Engineering International, Volume 2, No 1 (2014)

Table I: Thermo-power for Si $(660 \mu \mathrm{m})$ wafer

\begin{tabular}{|c|c|c|c|c|c|}
\hline \multicolumn{3}{|c|}{ n-type $(660 \mu \mathrm{m}) \mathrm{Si}$ wafer } & \multicolumn{3}{|c|}{ p-type $(660 \mu \mathrm{m}) \mathrm{Si}$ wafer } \\
\hline $\begin{array}{c}\text { Temperature } \\
\left({ }^{\circ} \mathrm{C}\right)\end{array}$ & $\mathrm{mV} /{ }^{\circ} \mathrm{C}$ & $\begin{array}{l}\text { Thermo- } \\
\text { power, } \mathrm{S}\end{array}$ & $\begin{array}{c}\text { Temperature } \\
\left({ }^{\circ} \mathrm{C}\right)\end{array}$ & $\mathrm{V} /{ }^{\circ} \mathrm{C}$ & $\begin{array}{l}\text { Thermo- } \\
\text { power, } \mathrm{S}\end{array}$ \\
\hline 50 & $8.75 / 50=0.175$ & \multirow{4}{*}{$\mathrm{S}=0.2$} & 50 & $0.76 / 50=0.015$ & \multirow{4}{*}{$S=0.02$} \\
\hline 60 & $10.5 / 60=0.175$ & & 60 & $0.99 / 60=0.0165$ & \\
\hline 70 & $15.3 / 70=0.219$ & & 70 & $1.6 / 70=0.022$ & \\
\hline 80 & $18.4 / 80=0.23$ & & 80 & $1.9 / 80=0.023$ & \\
\hline
\end{tabular}

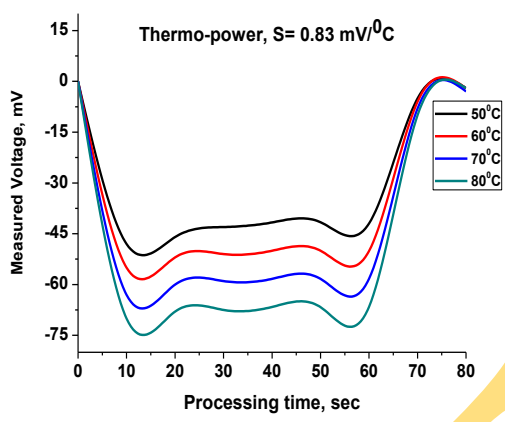

(a)

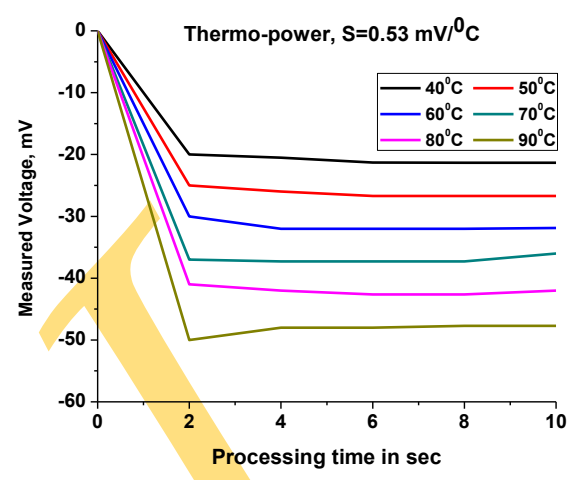

(b)

Figure 2. Characteristic curves for p-type commercial Si (200 $\mu \mathrm{m})$ sample. (a) Heating cycle curves (b) Cooling cycle curves

Table II: Thermo-power for commercial Si $(200 \mu \mathrm{m})$ wafer

\begin{tabular}{|c|c|c|c|c|c|}
\hline \multicolumn{3}{|c|}{ p-type Si wafer (Heating cycle) } & \multicolumn{3}{|c|}{ p-type Si wafer (Cooling cycle) } \\
\hline $\begin{array}{c}\text { Temperature } \\
\left({ }^{\circ} \mathrm{C}\right)\end{array}$ & $\mathrm{mV} /{ }^{\circ} \mathrm{C}$ & $\begin{array}{l}\text { Thermo- } \\
\text { power, S }\end{array}$ & $\begin{array}{c}\text { Temperature } \\
\left({ }^{\circ} \mathrm{C}\right)\end{array}$ & $\mathrm{mV} /{ }^{\circ} \mathrm{C}$ & $\begin{array}{l}\text { Thermo- } \\
\text { power, } S\end{array}$ \\
\hline 50 & $41.65 / 50=0.833$ & \multirow{6}{*}{$S=0.83$} & 40 & $21.32 / 40=0.53$ & \multirow{6}{*}{$S=0.53$} \\
\hline 60 & $50 / 60=0.833$ & & 50 & $26.7 / 50=0.53$ & \\
\hline 70 & $58.3 / 70=0.833$ & & 60 & $32 / 60=0.53$ & \\
\hline \multirow[t]{3}{*}{80} & $66.6 / 80=0.833$ & & 70 & $37.3 / 70=0.53$ & \\
\hline & $x_{3}$ & & 80 & $42.64 / 80=0.53$ & \\
\hline & & & 90 & $48 / 90=0.53$ & \\
\hline
\end{tabular}

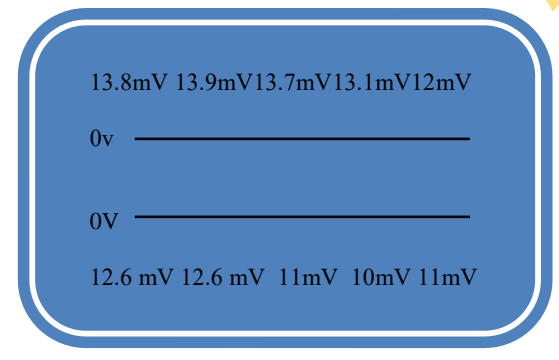

(a) Edge isolated front side

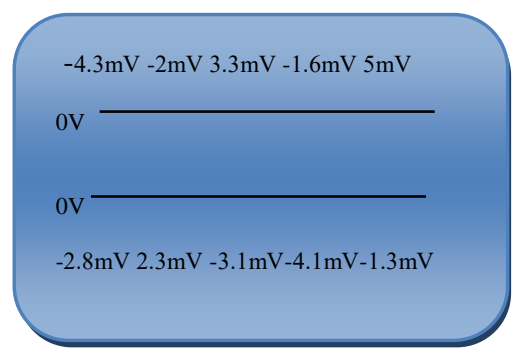

(b) Back side

Figure. 3 Wafer mapping for phosphorus doped commercial Si (200 $\mu \mathrm{m})$ wafer

Fig. 3 (a) shows that uniform phosphorus doping and the average potential difference $12.37 \mathrm{mV}$ through out the wafer front face. That means after doping emitter layer converted to $n$ type. Fig. 3 
(b) shows that non uniform doping at the rear side and the average potential difference $+/-2.98 \mathrm{mV}$ through out the wafer rear face.

\section{CONCLUSION}

An approach using low cost hot-probe method to monitor doping uniformity during solar cell fabrication process was successfully obtained. The measurements of the thermogenerated voltage between hot and cold electrodes along a heating and cooling cycle allow us to calculate Seebeck coefficient.

\section{FUTURE WORK}

In future, we will calculate the majority charge carrier concentration for Silicon sample by using the relationship between the initial state and steady state of the characteristic curves that would be compared with the conventional measurements.

\section{REFERENCES}

Alexander Axelevitch, Gady Golan (2013), "Hot-probe method for evaluation of majority charged carriers concentration in semiconductor thin films" Electronics and Energetics, vol. 26, no. 3, pp. $187-195$.

ASTM Standard F42-93, "Standard Test Methods for Conductivity Type of Extrinsic Semiconducting Materials," 1996 Annual Book of ASTM Standards, Am. Soc. Test. Mat. West Conshohocken, PA, 1996.

W.A. Keenan, C.P. Schneider and C.A. Pillus (1971), "Type-All System for Determining Semiconductor Conductivity Type," Solid State Technology, vol. 14, no. 3, pp. 51-56.

\section{Asian Business Consortium is an independent research house committed to publishing and delivering superior, Peer-reviewed standard research}

